\title{
Intra-aortic balloon pump for treatment of refractory ventricular tachycardia in Tako-Tsubo cardiomyopathy: A case report
}

\author{
Elisabetta Lisi ${ }^{\mathrm{a}, \mathrm{b}}$, Valentina Guida ${ }^{\mathrm{a}, \mathrm{b}}$, Simonetta Blengino ${ }^{\mathrm{a}}$, Elisabetta Pedrazzi ${ }^{\mathrm{a}}$, \\ Deborah Ossoli a , Gianfranco Parati ${ }^{\mathrm{a}, \mathrm{b}, *}$ \\ a Dept. of Cardiology, Istituto Auxologico Italiano, Milano, Italy \\ b Dept. of Health Sciences, University of Milano-Bicocca, Milano, Italy
}

\section{A R T I C L E I N F O}

Article history:

Received 28 January 2014

Accepted 14 March 2014

Available online 21 March 2014

\section{Keywords:}

Tako-tsubo

cardiomyopathy

arrhythmias

intra-aortic ballon pump

Tako-Tsubo cardiomyopathy (TTC) is characterized by a transient dysfunction of the left ventricular apex, often triggered by emotional or physical stress. Estimated prevalence of TTC ranges from 0.1 to $2.2 \%$ of patients with acute coronary syndrome (ACS). It usually occurs in old postmenopausal women. It often presents with chest pain and ECG changes (ST elevation in precordial leads and subsequent T wave inversion) and minimal myocardial enzymatic elevation which could mimic ACS, but in absence of coronary artery disease. Typical echocardiographic pattern shows apical-mid-ventricular akinesis and basal hyperkinesis. Acute heart failure and cardiogenic shock (CS) are the two most frequent TTC complications, but ventricular arrhythmias (VA) may also occur [1].

We report a case of TTC presenting as an incessant sustained ventricular tachycardia (VT).

A 81-year-old woman was brought to our emergency department with shortness of breath and chest pain. She had a history of hypertension under therapy with valsartan, amlodipine and clonidine, diabetes and hypercholesterolemia treated respectively with metformin and rosuvastatin. One year before, paroxysmal atrial fibrillation occurred and warfarin and amiodarone prophylaxis were introduced. On physical examination in emergency department the patient was conscious, with heart rate 110 beats/min and blood pressure 120 / $70 \mathrm{~mm} \mathrm{Hg}$. The electrocardiogram showed a wide-QRS tachycardia with left bundle branch block morphology compatible with sustained monomorphic VT (Fig. 1).

On laboratory tests serum troponin I was $0.91 \mathrm{ng} / \mathrm{mL}$, CKMB $9.1 \mathrm{ng} / \mathrm{mL}$ (normal values $<0.01 \mathrm{ng} / \mathrm{mL}$ and $<6.3 \mathrm{ng} / \mathrm{mL}$, respectively) and potassium $3.5 \mathrm{mmol} / \mathrm{L}$. The patient was initially treated with iv $\mathrm{MgSO}_{4}$ and potassium, since she was already under amiodarone therapy. Four ineffective electrical shocks were delivered.

In intensive care unit she was treated with iv lidocaine, diuretics, acetylsalycilic acid with persistence of VT. Overdrive transvenous temporary cardiac pacing wire was applied without benefit. Subsequently iv amiodarone was used with transitory restoration of sinus rhythm, then followed by multiple runs of sustained VT. Transthoracic echocardiography showed apical-mid-ventricular akinesis and basal hyperkinesis with left ventricular ejection fraction (LVEF) 30-35\%. No coronary stenosis was found on angiography. Imaging findings, mildly elevated troponin I level despite regional kinesis alterations and normal coronary angiogram were consistent with TTC diagnosis.

\footnotetext{
* Corresponding author at: Dept. of Cardiology, S.Luca Hospital, Istituto Auxologico Italiano \& University of Milan-Bicocca, Piazza Brescia 20, Milan, 20149-Italy. Tel.: +39 02619112949; fax: + 3902619112956.

E-mail address: gianfranco.parati@unimib.it (G. Parati).
}

The day after, due to persistence of sustained VT, low dose metoprolol was added and lidocaine was reintroduced with no benefit. After few hours we observed a rapid worsening of hemodynamic status consistent with CS. Therefore, upon orotracheal intubation, intra-aortic balloon pump (IABP) was placed, with prompt interruption of VT and progressive improvement of hemodynamic conditions. $24 \mathrm{~h}$ later levosimendan was added to support IABP weaning off.

At continuous ECG monitoring no VA were recorded and echocardiogram showed a progressive resolution of left ventricular (LV) contractile abnormalities with LVEF 63\%.

Some days later, ECG showed diffusely deep inverted T waves and QTc $500 \mathrm{~ms}$, so amiodarone was stopped. The patient was discharged with warfarin, lysine-acetylsalicylate $75 \mathrm{mg}$, ramipril $5 \mathrm{mg}$ bid, bisoprolol $2.5 \mathrm{mg}$ od, rosuvastatin $5 \mathrm{mg}$ od and amlodipine $5 \mathrm{mg}$ od.

Life-threatening VA are occasionally described as a TTC clinical manifestation, reported in $3.4 \%$ of cases and in $1.1 \%$ of cases as its first clinical presentation [2,3]. In spite of underlying sympathetic activation and frequently associated QTc prolongation [3], VA seem to be less common than expected in TTC, although they might represent an important component of this syndrome. Indeed, QTc prolongation associated with autonomic dysregulation in TTC is recognized as important risk factor for VT [2]. As shown in Wittstein's paper [4] in TTC patients plasma catecholamine levels are two-to-threefold increased as compared with ACS and LV failure patients. However the possible mechanisms underlying myocardial stunning in TTC are controversial and could include epicardial coronary spasm, microvascular dysfunction and direct catecholamine induced myocyte injury. As TTC typical contractile abnormalities are localized in multiple vascular territories, the epicardial spasm hypothesis seems unlikely; whereas both microvascular spasm, suggesting sympathetically mediated microcirculatory dysfunction, and direct myocyte injury, may be involved in myocardial stunning [4]. Microvascular dysfunction can lead to an oxygen demand impairment that could increase myocardial vulnerability and create the ideal substrate for VA. In Gianni's metanalysis CS occurred in $4.2 \%$ of TTC patients and almost $10 \%$ of them needed IABP [5]; similarly in Sharkey's paper about 30\% of TTC patients with CS required inotropes or mechanical circulation support [6]. Catecholamine inotropes are often used in the treatment of CS. However, since catecholamine surge seems to be implicated in TTC pathogenesis, inotrope use is controversial. As described in isolated case-reports, only a non-catecholamine inotrope, such as levosimendan, might be beneficial in TTC patients with CS [7].

In this clinical context even the use of IABP support is still debated. Thanks to its property to improve myocardial perfusion and reduce afterload and myocardial oxygen consumption, IABP can be used in patients with CS after myocardial infarction. Albeit latest international ST elevation myocardial infarction (STEMI) guidelines [8] downgrade IABP use in CS after STEMI from class I to class II, IABP seems to be effective in stabilizing patients with refractory VA after myocardial infarction by increasing coronary perfusion pressure, reducing transmyocardial wall stress and maintaining adequate systemic perfusion [9].

On the contrary, there are only few reports about IAPB use in CS patients with normal coronary arteries [10] and even less is available about its use in TTC, especially when CS caused by VA occurs. In our case for the first time we described a successful IABP use in a TTC patient 


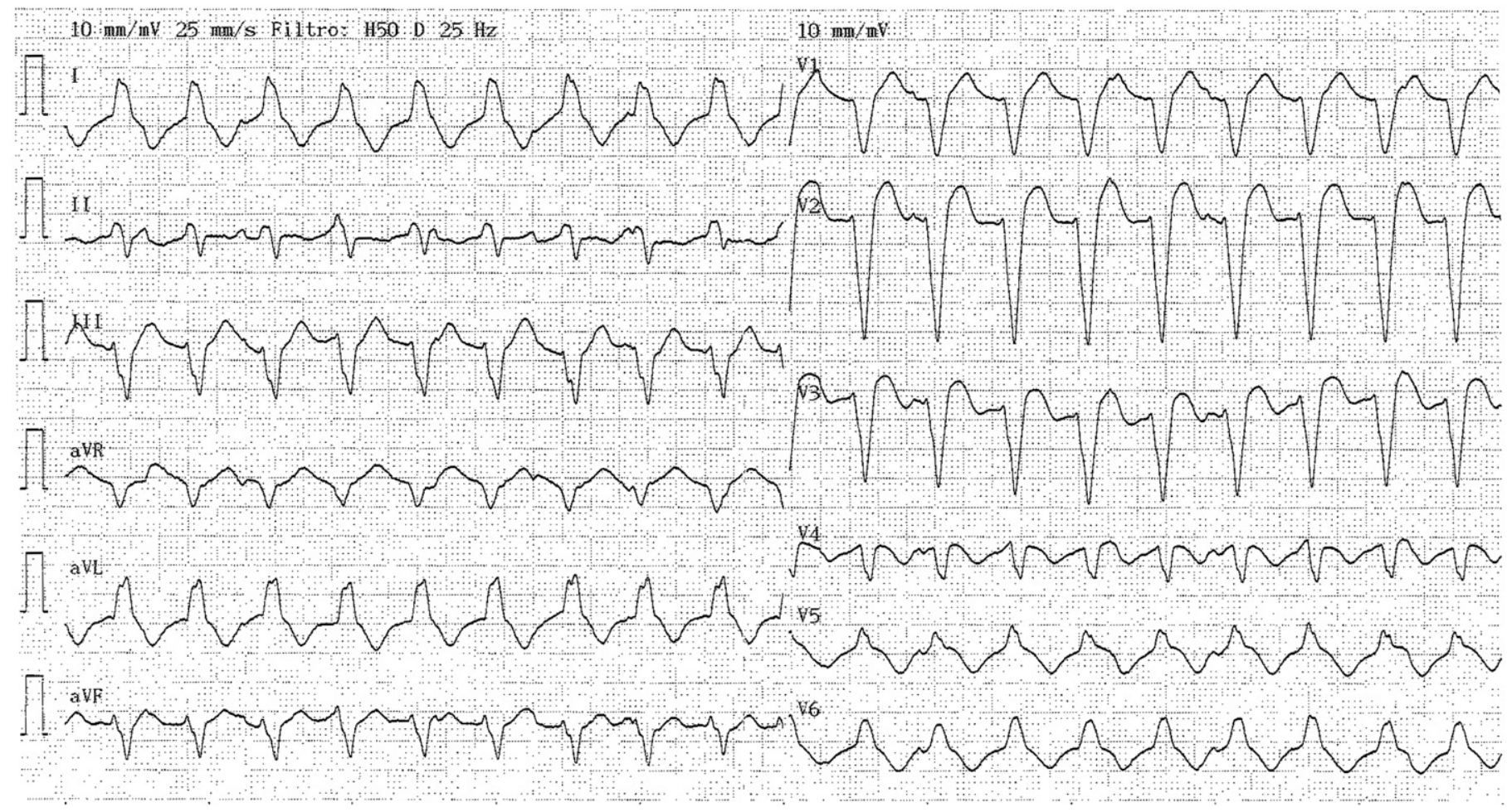

Fig. 1. Electrocardiogram in emergency room: it shows a wide-QRS tachycardia with left bundle branch block morphology compatible with monomorphic VT.

with hemodynamic instability due to VT refractory to conventional antiarrhythmic therapy. After IABP application, we observed a quick resolution of VA, followed by a progressive LV function improvement.

In conclusion, VT can be more frequent than expected lifethreatening presentation of TTC. There is no clear evidence about its best treatment in TTC patients, especially when conventional antiarrhythmic procedures failed. In particular, while IABP has been reported to effectively manage refractory VT in ischemic patients, no evidence is available on its usefulness in non-ischemic refractory VT. Our case report suggests that IABP may be considered as support treatment in refractory non-ischemic VT, especially when hemodynamic impairment occurs. Our observation should thus stimulate additional studies aimed to systematically test this possible therapeutic approach.

\section{References}

[1] Kono T, Sabbah HN. Takotsubo cardiomyopathy. Heart Fail Rev 2013, doi:10.1007/ s10741-013-9404-9.
[2] Hasdemir C, Vuran O, Yuksel A, Yavuzgil O. Stress cardiomyopathy (Tako-Tsubo) associated with sustained polymorphic ventricular tachycardia. Pacing Clin Electrophysiol 2013;36:111-4.

[3] Syed FF, Asirvatham SJ, Francis J. Arrhythmia occurrence with takotsubo cardiomyopathy: a literature review. Europace 2011;13:780-8.

[4] Wittstein IS, Thiemann DR, Lima JA, et al. Neurohumoral features of myocardial stunning due to sudden emotional stress. N Engl J Med 2005:539-48.

[5] Gianni M, Dentali F, Grandi AM, Sumner G, Hiralal R, Lonn E. Apical ballooning syndrome or takotsubo cardiomyopathy: a systematic review. Eur Heart 2006;27:1523-9.

[6] Sharkey SW, Lesser JR, Zenovich AG, et al. Acute and reversible cardiomyopathy provoked by stress in women from the United States. Circulation 2005;111:472-9.

[7] Padayachee L. Levosimendan: the inotrope of choice in cardiogenic shock secondary to takotsubo cardiomyopathy? Heart Lung Circ 2007;16(Suppl 3): S65-70.

[8] O'Gara PT, Kushner FG, Ascheim DD, et al. 2013 ACCF/AHA guideline for the management of ST-elevation myocardial infarction: a report of the American College of Cardiology Foundation/American Heart Association Task Force on Practice Guidelines. J Am Coll Cardiol 2013;61(4):e78-e140.

[9] Stone GW, Ohman EM, Miller MF, et al. Contemporary utilization and outcomes of intra-aortic balloon counterpulsation in acute myocardial infarction: the benchmark registry. J Am Coll Cardiol 2003;41(11):1940-5.

[10] Fotopoulos GD, Mason MJ, Walker S, et al. Stabilisation of medically refractory ventricular arrhythmia by intra-aortic balloon counterpulsation. Heart 1999;82(1):96-100 